\title{
TRAFFIC DENSITY MONITORING AND CATTLE MENACE ALERT SYSTEM USING IOT
}

\author{
Rashmi P. Nimkar ${ }^{1}$, Chandrashekhar N. Deshmukh ${ }^{2}$ \\ ${ }^{I}$ Student, M.E, Electronics \& Telecommunication Engineering, Prof. Ram Meghe Institute of Technology \& Research, \\ Badnera-Amravati, Maharashtra, India \\ ${ }^{2}$ Associate Professor, Electronics \& Telecommunication Engineering, Prof. Ram Meghe Institute of Technology \& \\ Research, Badnera-Amravati, Maharashtra, India
}

\begin{abstract}
In today's Life we have to face one serious problem of traffic congestion in modern cities. The main reasons for increasing traffic jam is the high volume of vehicles and the inadequate infrastructure. It is necessary to efficiently manage the traffic flow by completely utilizing the existing capacity of the road. Another serious problem is the presence of animals on the road. Stray cattle blocking the driveway triggers collisions and accidents. A Smart traffic control system can solve these problems by continuously detecting and adjusting the timing of traffic signals according to the actual traffic load such a system called as intelligent traffic control system. In the era of technology, intelligent and adaptive equipment should be used to control the traffic. In this paper we proposed a Traffic Density Monitoring and Cattle Menace Alert System Using IoT. This smart traffic monitoring and controlling system is use to decrease the number of traffic-jam, vacating the road which has more density of vehicles by synchronizing the traffic signals as well as to develop an alert system once the animal gets detected. The proposed system uses a concept of Internet Of Things application platform 'ThingSpeak' for analysis of traffic density monitoring.
\end{abstract}

Keywords: - Traffic Congestion, Image Processing, Internet of Things, Thingspeak, Cattle Detection

\section{INTRODUCTION}

One of the daily routine activities for most people is travelling. To travel to different places within the city commuters use different type of vehicles, car, bus, motorbike etc. Roads and vehicular traffic are essential part of the day-to-day life of the people. Congestion in traffic is a serious problem nowadays. Traffic congestion problems consist of incremental delay, vehicle operating costs such as fuel consumption, pollution emissions and stress that result from interference among vehicles in the traffic stream. Various studies reveal that the road traffic noise is the major contributor of noise pollution in the urban areas, Hence, analyzing and monitoring the traffic patterns is done ceaselessly to improve traffic conditions. One another serious problem is the presence of animals on the road. Stray cattle roaming around freely in the cities causing traffic blockheads and accidents on city roads are increasing day by day. Therefore the detection of cattle is required in traffic monitoring system.

The goal of this current research is to develop an automatic vehicle counting system, which can process videos recorded from stationary cameras over roads e.g. CCTV cameras installed near traffic intersections / junctions and counting the number of vehicles passing a spot in a particular time for further collection of vehicle / animals traffic data. The paper aims to automate the traffic control system on junctions to determine Traffic Density in order to count the cars and monitoring of roads. This smart traffic monitoring and controlling system is use to decrease the number of trafficjam, vacating the road which has more density of vehicles by synchronizing the traffic signals as well as to develop an alert system once the animal gets detected on the road. The analysis of traffic monitoring will be done using Thingspeak Channel. ThingSpeak is an IoT analytics platform, having capabilities of real-time data collection and visualizing the collected data in the form of charts.

\section{EXISTING METHODS}

Many researches and works have been done on traffic For management and controlling of traffic several researches have been conducted using image processing, embedded system, wireless sensor networks, RFID,GSM technology and Artificial Intelligence Techniques. In [1] development of a self-adaptive system is presented which can help in better traffic management using the technique of image processing. Using image matching, reference image of an empty road and the captured images are sequentially matched. Here Prewitt edge detection operator is used for carrying edge detection and according to percentage of matching traffic light durations can be controlled.

An approach for analysis and detecting vehicles in highways traffic images by means of image processing techniques such as background differencing, Otsu's thresholding and morphological filters is presented in [2]. Then Region and image Properties are used to count the number of objects in an image. Harshini Vijetha H, Dr. Nataraj K R. [3], 
presented a new approach for controlling Traffic System .In the proposed system with components like Raspberry Pi, PiCamera, RFID, IR sensors an intelligent traffic controller is designed. In this paper with the help of IR sensors density of the traffic will be decided. Using RFID technology, Green path (Zero traffic) is given to emergency vehicles and trailing of the stolen automobiles is also done.

In [4], the solutions for Traffic congestions using the concept of networking and embedded systems are explained. Author designed a alert system using Raspberry Pi, Routers, Ultrasonic sensor and E-Mail servers. The author in [5] proposed Road Traffic Congestion Observing and Measurement using Active RFID and GSM Technology. To monitor traffic and detect congestion, author developed a Traffic Monitor system makes use of RFID enabled probe vehicles. This system use roadside active wireless devices to collect signals from active RFID tags attached to the probe vehicle. The implemented system trace the travel time of probe vehicle as it passes the roadside devices, create an average trip time.

In [6], in order to elicit general diagnosis for the traffic congestion problem in Kuala Lumpur and Kuantan, author presented a new Artificial Intelligence Techniques (AIT) and Simulation Model (SM),. Author developed a model which involves a Neural Network (NN), Fuzzy Logic (FL), Genetic Algorithm (GA), and Simulation Model (SM) In [7] ,an advanced traffic management system, implemented using Internet of Things (IoT) was developed. Here embedded circuit which operates using RFID with clustered systems is used the vehicle,. For working with big data analytics, Hadoop is presented. Here supervised learning methodologies are proposed that would help in determining the standard of roads, estimating overall traffic flow, calculating average speed of distinct vehicle types on a road and analyzing the travel path of a vehicle.

Different techniques for detection and tracking of animals have been used by many researchers.

In [8] author proposed WSN based system for wildlife management in the surrounding area of human passages to establish safe ways for animals to cross transportation infrastructures. The detector circuit is designed using pirbased motion detectors. These detectors are used to detect the presence of an animal. In [9], for animal detection author discussed animal detection using viola and jone algorithm. In this paper, videos are captured by camera and converted into frames. After finding the different images, a database for Positive and negative images is created. In Positive images correspond to image with detected animal and negative images correspond to image with non detected animal. HAAR Transform is used for feature extraction. In [10], to detect the animal presence using the web camera and for identification of dangerous animal a animal detection system is proposed. If the animal is wild, the safety automatic animal detection and warning system is developed to warn the people. Here for object detection CBRA (i.e. Content Based Retrieval Algorithm) is used.

However, all the above mentioned existing methods in the literature uses image processing techniques, embedded systems, IoT and Artificial Intelligence Techniques, each have their own pros and cons. The existing methods are tedious and require large number of hardware. In the above section we have discussed the multiple researches method which process images and track vehicles and focus on improving the traffic conditions and cattle menace detection. They are still too complex which demand high computational processing. To reduce the cost and optimize the solution, proposed system uses both image processing techniques and Iot analytic platform "Thingspeak" for monitoring traffic density and cattle menace detection.

\section{DESIGN OF TRAFFIC DENSITY MONITORING AND CATTLE MENACE ALERT SYSTEM USING IOT}

To have better traffic control, by utilizing image processing and IoT application platform "ThingSpeak" a new model is developed. This system consists of video cameras on the traffic junction, capturing video and broadcasting it to the server where using video and image processing techniques the vehicle density on the road is calculated. After getting a brief view related to traffic condition, total number of vehicles will be calculated and presence of animal on the road will be detected by image processing from CCTV Camera. MATLAB programming environment will be used for developing the proposed system. The analysis of traffic monitoring would be done using Thingspeak Channel.

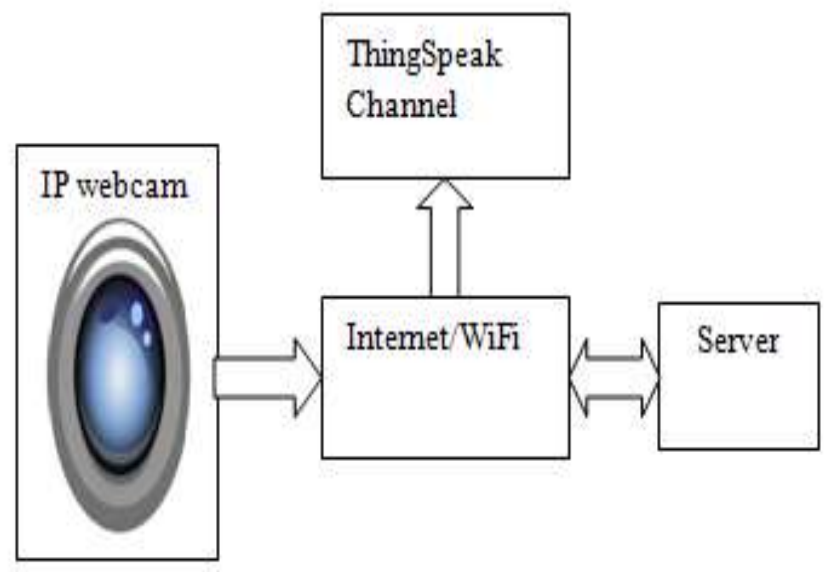

Fig 1: Main Block diagram of proposed system

According to Main block diagram, the data related to traffic will be captured using IP web camera. Using internet videos related to traffic are sent to computer. The videos which are captured by the camera will be processed here. Using internet the vehicle density count is uploaded ON ThingSpeak channel for analysis. 


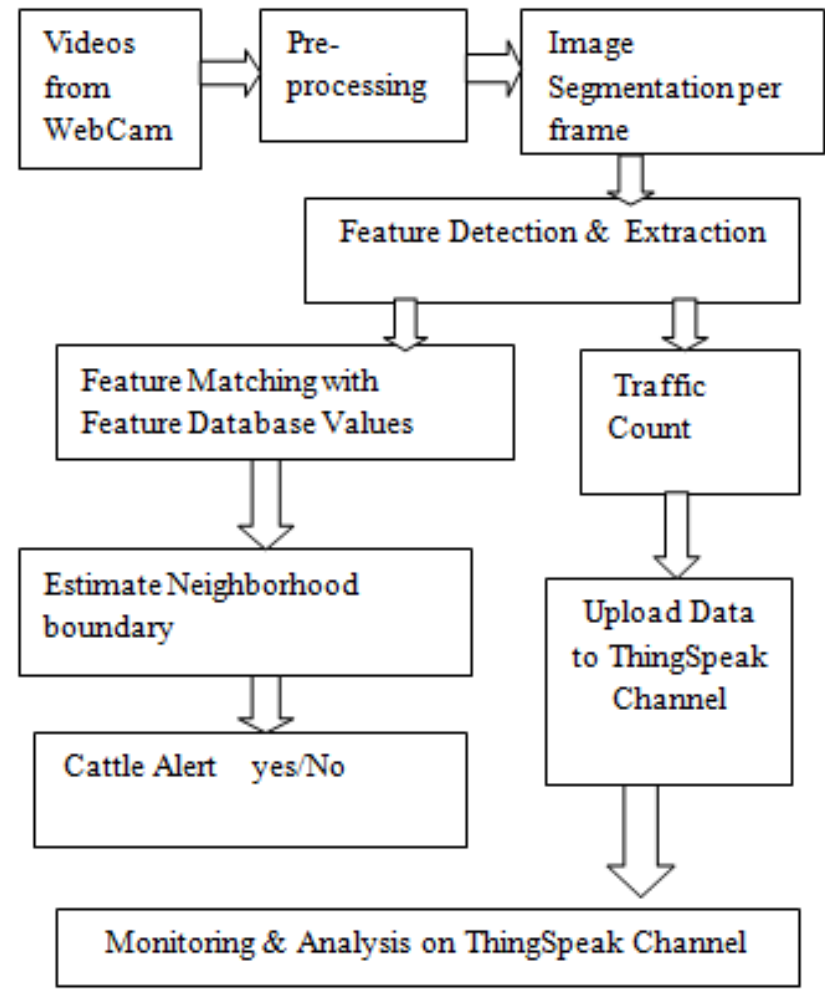

Fig 2: Server system

In server these videos are splits in the form of frames. The block diagram of server system consists of two parts: 1.TrafficMonitoring System 2.Cattle Detection And Alert System

\subsection{Traffic Monitoring System}

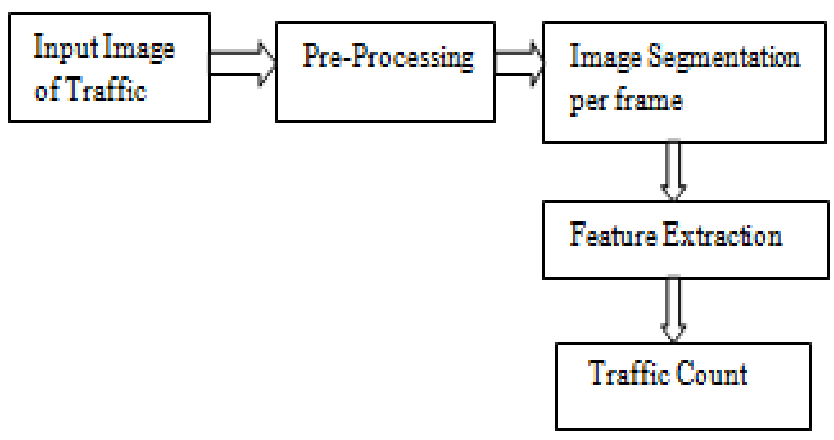

Fig 3: Block diagram of Traffic Monitoring system

Obtained frames are processed from following phases: 1 . Pre-Processing Phase 2. Image segmentation. The aim of pre-processing is suppresses unwanted distortions or enhances some image features important for further processing. For noise removal we use median filter. This filter belongs to the class of edge preserving smoothening filters which are non-linear filters. This means that for two images $\mathrm{A}(\mathrm{x})$ and $\mathrm{B}(\mathrm{x})$ :

$$
\operatorname{median}[\mathrm{A}(\mathrm{x})+\mathrm{B}(\mathrm{x})] \neq \operatorname{median}[\mathrm{A}(\mathrm{x})]+\operatorname{median}[(\mathrm{x})]
$$

These filters smoothes the data while keeping the small and sharp details. The median is just the middle value of all the values of the pixels in the neighborhood. The median filter considers each pixel in the image in turn and looks at its nearby neighbors to decide whether or not it is representative of its surroundings. The pixel value replaces it with the median of those values. The median is calculated by first sorting all the pixel values from the surrounding neighborhood into numerical order and then replacing the pixel being considered with the middle pixel value.

For detecting moving objects in videos from static cameras Background subtraction is used. Background subtraction, also known as foreground detection, is a technique in the fields of image processing and computer vision wherein an image's foreground is extracted for further processing. To determine whether individual pixels are part of the background or the foreground, the foreground detector system object compares a color or grayscale video frame to a background model. It then computes a foreground mask using Gaussian mixture models.

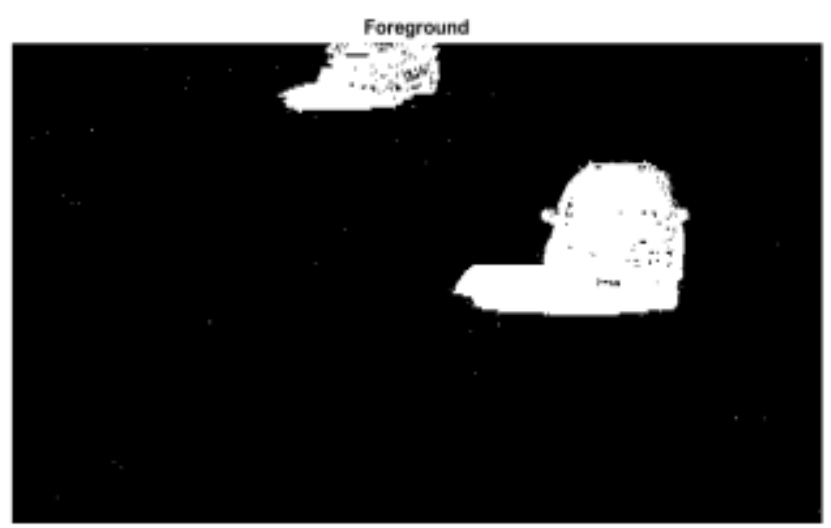

Fig 4: The foreground mask computed by the detector

Segmentation or contouring could be also obtained using morphological operations. Segmentation subdivides an image into its constituent regions or objects. To detect the shape of the object, the segmented image undergoes a series of morphological operations. Morphological image processing is a collection of non-linear operations related to the shape or morphology of features in an image. Dilation and erosion are basic morphological processing operations. Both dilation and erosion are produced by the interaction of a set called a structuring element with a set of pixels of interest in the image. We have used the strel function to create a flat structuring element. A strel object represents a flat morphological structuring element. It is an essential part of morphological dilation and erosion operation. A flat structuring element is a binary valued neighborhood, either 2-D or multidimensional, in which the true pixels are included in the morphological computation, and the false pixels are not. The center pixel of the structuring element, 
called the origin, identifies the pixel in the image being processed. Opening consists of an erosion followed by a dilation and can be used to eliminate all pixels in regions that are too small to contain the structuring element. Closing consists of a dilation followed by erosion and can be used to fill in holes and small gaps. Figure 5 shows morphological opening to remove the noise and to fill gaps in the detected objects.

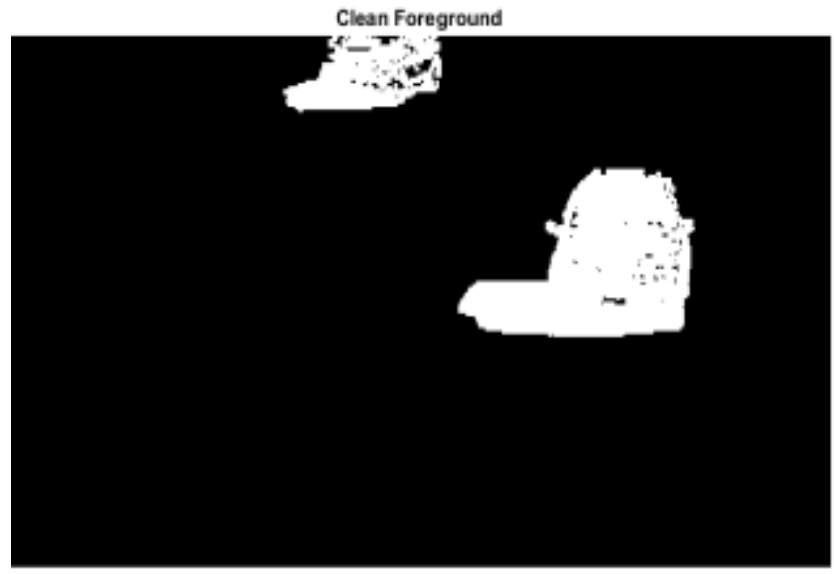

Fig 5: Output from morphological operation

For feature extraction blob analysis is used. The Blob Analysis object computes statistics for connected regions in a binary image. Region and image Properties are used to analyze the number of objects in an image. To get region properties information about the objects in an image, centroid of the detected vehicle is computed. Using centroid points, number of objects is counted and displayed. We found bounding boxes of each connected component corresponding to a moving car by using vision.BlobAnalysis object. Here we found the number of bounding boxes corresponds to the number of cars found in the video frame as shown in Fig 6.

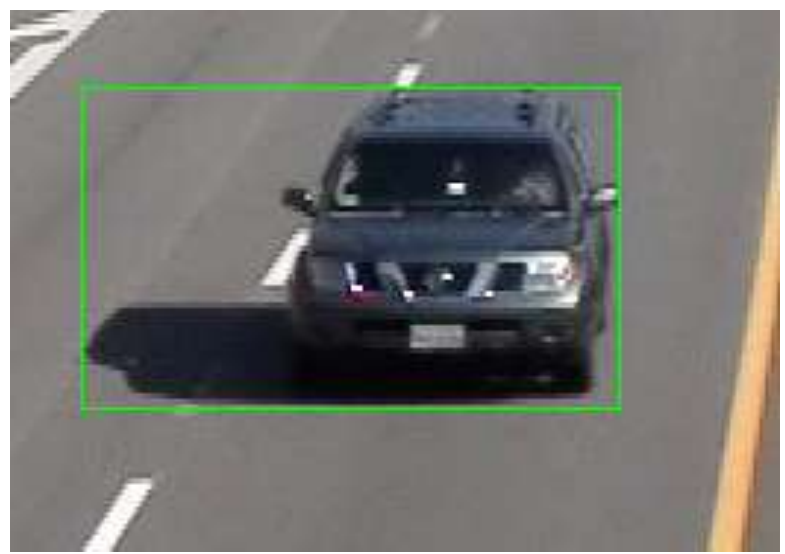

Fig 6: Output from vision.BlobAnalysis object

The number of found cars is displayed in the upper left corner of the processed video frame. Here we got the number of cars found in the video frame and Set the timer according to vehicle density for traffic light controlling. Upload this vehicle density count on ThingSpeak Channel. To use ThingSpeak, we need to signup and create a channel. Once we have a channel, we can send the data, allow ThingSpeak to process it and also retrieve the same.

\subsection{Cattle Detection and Alert System}

For cattle detection, we collect some images of cattle to create database for cattle. The database is then split into a training dataset and a testing dataset.

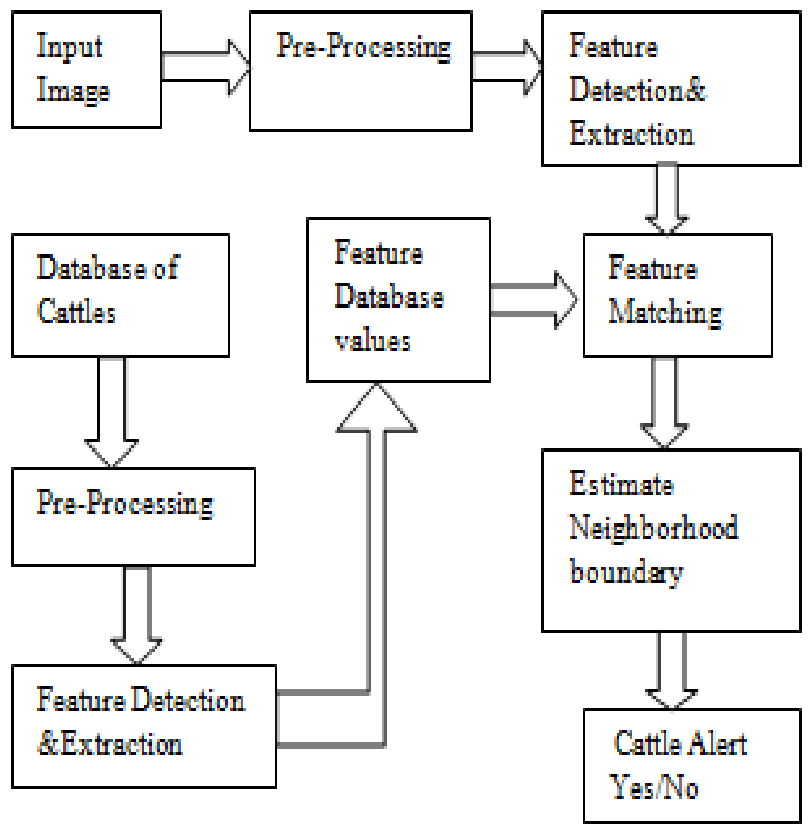

Fig 7: Block diagram for cattle detection and alert system

For cattle detection, after preprocessing and Image segmentation Feature extraction is done. For Feature extraction HOG (Histogram of Oriented Gradients) is used. The histogram of oriented gradients (HOG) is a feature descriptor used in computer vision and image processing for the purpose of object detection. The HOG descriptor technique counts occurrences of gradient orientation in localized portions of an image -detection window, or region of interest (ROI). Once features have been extracted, feature matching is done .For feature matching KNN classifier is used. The training phase of the KNN algorithm consists only of storing the feature vector and class labels of the training samples. In the classification phase, $k$ is a user-defined constant, and an unlabeled vector (a query or test point) is classified by assigning the label which is most frequent among the $k$ training samples nearest to that query point. Euclidean distance is commonly used distance metric for continuous variable. 


\section{Steps for Cattle Detection}

Training stage:

1. Load Data Base: First load the data from the training data base for training purpose

2. Extraction Data Base Feature: Extract the training data set feature

3. Save Features and its Classes : Save the extracted feature of training data set

Testing Stage:

1. Load Input Data: In testing stage at first load the input data which is to be test.

2. Extract Features: After loading the testing data extract its feature for classification.

3. Evaluate: After feature extraction, evaluate the data using feature matching process for cattle detection

The output after training and testing stage is shown in Fig,8.

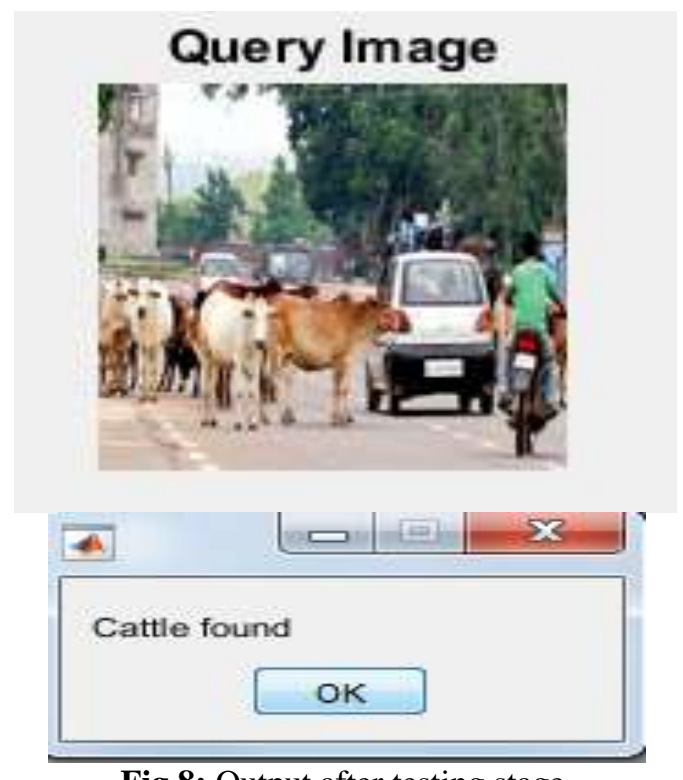

Fig 8: Output after testing stage

After testing stage, a alert message "cattle found" is displayed when the cattle is detected.

\section{RESULTS AND DISCUSSION}

Hardware used for Traffic Monitoring and Cattle menace detection system is personal computer with 64 bit Windows 10 Pro operating system with MATLAB R2016a 64 bit version. The data is collected from videos recorded by IP web cam. In this work the background segmentation algorithm, noise reduction through morphological analysis, blob detection and signal system based on number of blobs or density a module have been developed. Process starts with reading a input frame, we convert this RGB into gray

images. Applying background subtraction algorithm with noise Reduction process, we find the number of vehicles in the road. Number of blobs is the actual density of vehicle and according to vehicle density on road Traffic light timer changes. This vehicle density count is uploaded on ThingSpeak channel by signing up and setting up a channel. As per the vehicle count the traffic light timer is set. The proposed method tested on 11 videos captured using IP webcam. Table1 presents the videos used in the experiments indicating date and time in the video name itself and video parameters. The performance of the traffic monitoring system is shown in Table 2. Some of images for traffic density count are shown in following Fig 9.

\subsection{For Traffic Density Count}

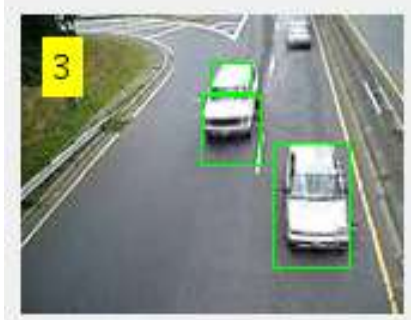

Image 1

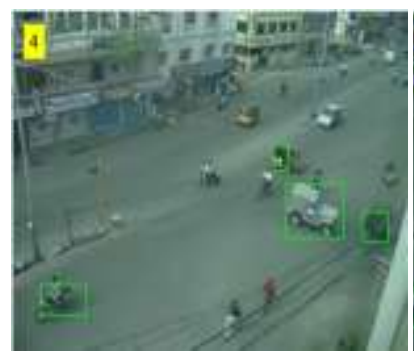

Image 3

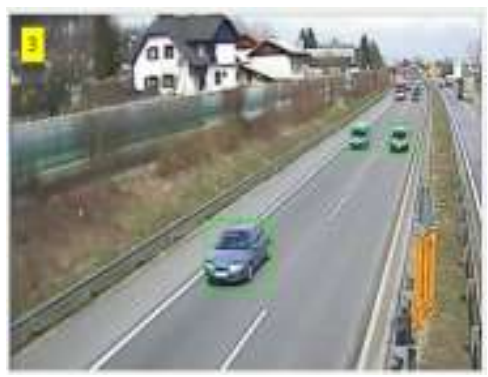

Image 5

Fig 9:e Images 1, 2, 3, 4 and 5 shows the detected and counted vehicles 
Table 1: Video sequences used in our experiments

\begin{tabular}{|l|l|l|l|l|l|}
\hline Sr.No & Teat case videos & \multicolumn{2}{l|}{ Video Parameters } \\
\hline & & Frame Rate F/S & Width & Height & Duration \\
\hline 1 & 22-May-2018_080225_record.avi & 25 & 380 & 214 & $30 \mathrm{sec}$ \\
\hline 2 & 22-May-2018_080255_record.avi & 25 & 380 & 214 & $30 \mathrm{sec}$ \\
\hline 3 & 22-May-2018_080325_record.avi & 14 & 320 & 240 & $30 \mathrm{sec}$ \\
\hline 4 & 22-May-2018_080355_record.avi & 14 & 320 & 240 & $30 \mathrm{sec}$ \\
\hline 5 & 22-May-2018_080454_record.avi & 14 & 320 & 240 & $30 \mathrm{sec}$ \\
\hline 6 & 22-May-2018_080525_record.avi & 15 & 160 & 120 & $30 \mathrm{sec}$ \\
\hline 7 & 22-May-2018_080555_record.avi & 14 & 320 & 240 & $30 \mathrm{sec}$ \\
\hline 8 & 22-May-2018_080625_record.avi & 25 & 380 & 214 & $30 \mathrm{sec}$ \\
\hline 9 & 22-May-2018_080655_record.avi & 25 & 380 & 214 & $30 \mathrm{sec}$ \\
\hline 10 & 22-May-2018_080725_record.avi & 24 & 380 & 214 & $30 \mathrm{sec}$ \\
\hline 11 & 22-May-2018_080755_record.avi & 14 & 320 & 240 & $30 \mathrm{sec}$ \\
\hline
\end{tabular}

Table 2: Performance of the Traffic Monitoring system

\begin{tabular}{|l|l|l|l|l|l|l|}
\hline Sr.No & Teat case videos & MC & AC & EP & EN & Accuracy in \% \\
\hline 1 & 22-May-2018_080225_record.avi & 0 & 0 & 0 & 0 & 100 \\
\hline 2 & 22-May-2018_080255_record.avi & 1 & 1 & 0 & 0 & 100 \\
\hline 3 & 22-May-2018_080325_record.avi & 5 & 4 & 1 & 0 & 80 \\
\hline 4 & 22-May-2018_080355_record.avi & 3 & 5 & 0 & 2 & 60 \\
\hline 5 & 22-May-2018_080454_record.avi & 8 & 7 & 1 & 0 & 87.5 \\
\hline 6 & 22-May-2018_080525_record.avi & 2 & 2 & 0 & 0 & 100 \\
\hline 7 & 22-May-2018_080555_record.avi & 9 & 8 & 1 & 0 & 88.8 \\
\hline 8 & 22-May-2018_080625_record.avi & 2 & 2 & 0 & 0 & 100 \\
\hline 9 & 22-May-2018_080655_record.avi & 1 & 1 & 0 & 0 & 100 \\
\hline 10 & 22-May-2018_080725_record.avi & 1 & 1 & 0 & 0 & 100 \\
\hline 11 & 22-May-2018_080755_record.avi & 3 & 3 & 0 & 0 & 100 \\
\hline & & Average & & & 92.39 \\
\hline
\end{tabular}

- MC- Manual Count

- AC- Algorithmic Count

- EP- Error Positive

- EN- Error negative

\subsection{For Cattle Detection}

We have taken some different real time videos and find the output with the detected animal, result is shown in following figures with output alert message display "cattle found" or " No cattle found. Fig 10. shows the detected animals in the frame.

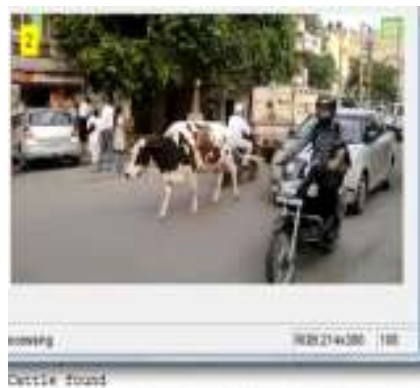

Image 1

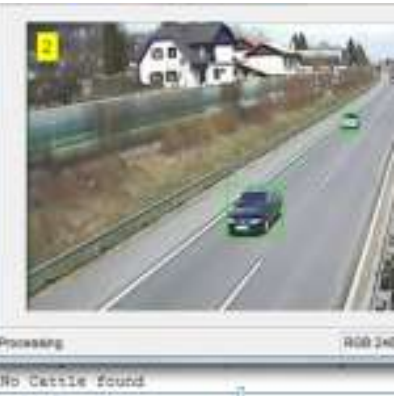

Image 2

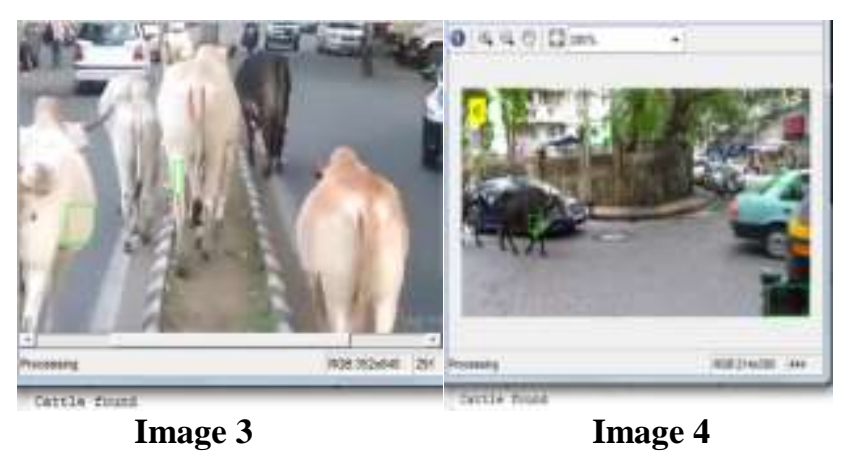

Fig 10: Images 1, 3, 4 shows the detected cattle

\subsection{Traffic Density Monitoring on ThingSpeak Channel}

The vehicle density count is uploaded on ThingSpeak channel by signing up on https://thingspeak.com/ and setting up a channel on ThingSpeak platform. 

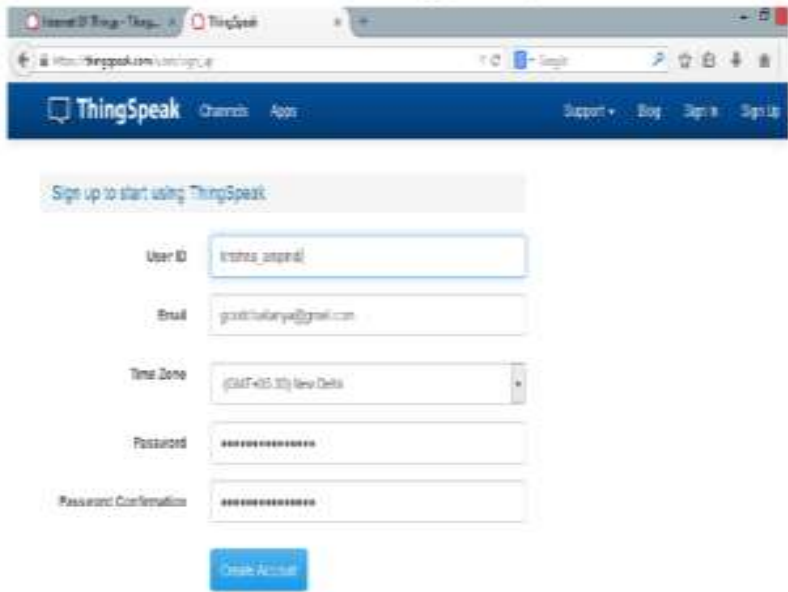

Fig 11: ThingSpeak Sign up window

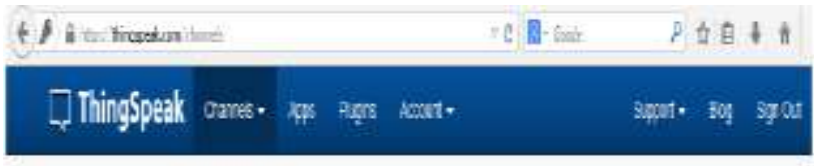
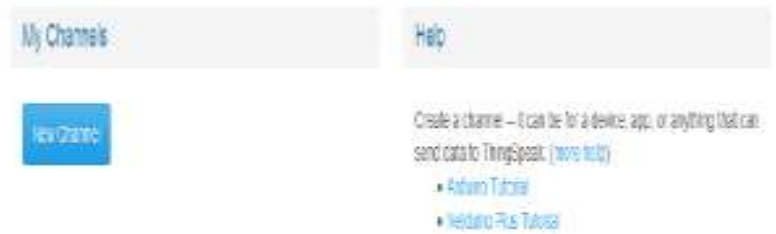

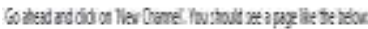

Fig 12: Creating Channel

In channel setting we added a description corresponding to the channel and a field before it can be used to store data. When we create a channel, by default, a write API key is generated. The write API key is used for sending data to the channel. A channel stores the data that we send to ThingSpeak

Figure 13 shows the traffic density monitoring analysis on ThingSpeak channel with current date and time. Each of the dots corresponds to the traffic density value and the time at which the traffic density value was posted to the channel.

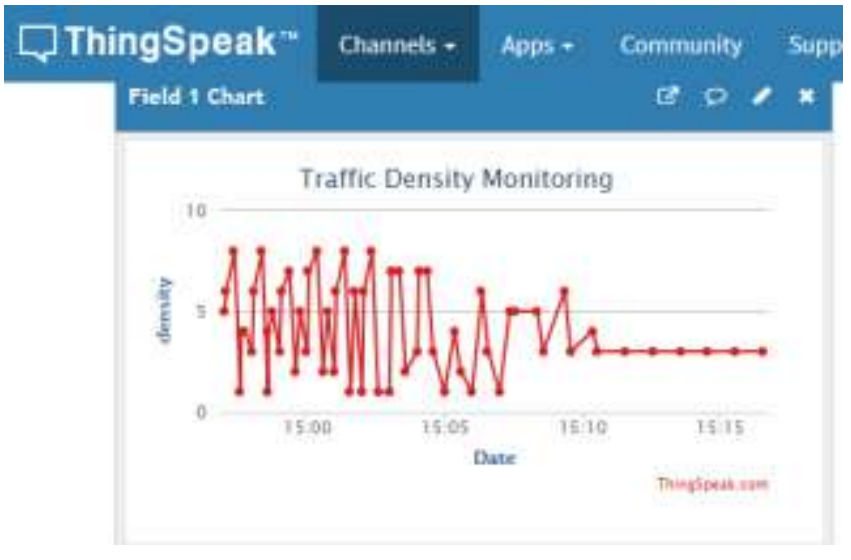

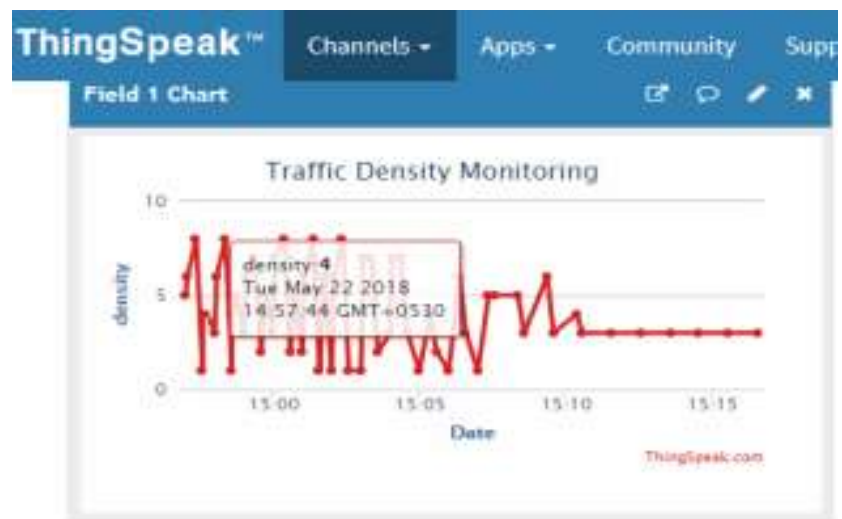

Fig 13: ThingSpeak Channel

\section{CONCLUSION}

The proposed method tested on 11 videos captured using IP webcam and the vehicle count accuracy average is $92.39 \%$. Also the cattle detection algorithm gives accurate results in less time for detection of cattle. On ThingSpeak channel the charts in the private view tab shows dots correspond to the traffic density value and the time at which the traffic density value was posted to the channel. In this paper by using IOT analytic platform we proposed a system for detecting the density of vehicles, cattle menace and processing the duration of the traffic signal. The proposed system is used for controlling the traffic, avoiding traffic congestion. Also, the signals are monitored and the status of the traffic signal is updated at the server. Cattle menace is detected. The proposed system is very cost effective as it does not require installation of any additional devices, such as RFIDs, sensors etc .Because this method use Image Processing, Matlab software and ThingSpeak platform, it is feasible to implement it with low cost and with optimum accuracy.

Using the proposed method we are only monitoring the number of vehicles present at the signal. This work can be enhanced further to detect the vehicles which disobey the traffic laws. This work can be enhanced further to identify the presence of emergency vehicles (like an ambulance or fire ) giving preference to those emergency vehicles. We can also use this method for garage management systems, for smoother parking of cars and vehicles

\section{ACKNOWLEDGEMENT}

The authors wish to thank all teaching and non teaching staff of Prof,Ram Meghe Institute of Technology \& Research for their consistent support.

\section{REFERENCES}

[1] Bhakti Viramgama, Prof. Hetal Chauhan," An Application of Image Processing: Traffic Management" e-ISSN (O): 2348-4470 p-ISSN (P): 2348-6406, June -2016 
[2] R. Sofia Janet, J. Bagyamani ,'Traffic Analysis on Highways based on Image Processing”, International Journal of Computational Intelligence and Informatics, Vol. 5: No. 1, June 2015

[3] Harshini Vijetha H, Dr. Nataraj K R," IOT Based Intelligent Traffic Control System", Volume 5 Issue V, May 2017IC Value: 45.98 ISSN: 2321-9653.

[4] Keertikumar B Malagund, Shubham N Mahalank, R.M.Banakar," IoT based smart city traffic alert system design"

[5] Koushik Mandal, Arindam Sen, Abhijnan Chakraborty and Siuli Roy," Road Traffic Congestion Monitoring and Measurement using Active RFID and GSM Technology", 2011 14th International IEEE Conference on Intelligent Transportation Systems Washington, DC, USA. October 5-7, 2011

[6] Khaled Abdul Rahman Jomaa,"An Artificial Intelligence Techniques and Simulation Model to Control a Traffic Jam System in Malaysia (Review Paper)"Asian Journal of Business and Management (ISSN: 2321 - 2802)Volume 04- Issue 01, February 2016

[7] Mahesh Lakshminarasimhan," IoT Based Traffic Management System "https://www.researchgate.net/publication/310036684 ,March 2016

[8] Nidhi Daxini , Sachin Sharma , Rahul Patel," Real Time Animal Detection System using HAAR Like Feature", International Journal of Innovative Research in Computerand Communication Engineering(An ISO 3297: 2007 Certified Organization) Vol. 3, Issue 6, June 2015

[9] S. Sharma, D. Shah," A Practical Animal Detection and Collision Avoidance System Using Computer Vision Technique" 2169-3536 (c) 2016

[10] Sajid Shaikh, Mayur Jadhav,Naveen Nehe and Prof. Usha Verma,",Automatic Animal Detection And Warning System", International Journal of Advance Foundation and Research in Computer (IJAFRC)Volume 2, Special Issue (NCRTIT 2015), January 2015. ISSN 2348 - 4853

[11] Tania Chawla, Dr Asmita A. Moghe, "Real-Time Traffic Detection using Horizontal and Vertical Scanning” ISSN: 2277-128X (Volume-7, Issue6)Research June2017

[12] Busarin Eamthanaku, Mahasak Ketcham, Narumol Chumuang," The Traffic Congestion Investigating System by Image Processing from CCTV Camera"

[13] Jiandong Cao,"'Research on Urban Intelligent Traffic Monitoring System Based on Video Image Processing" International Journal of Signal Processing, Image Processing and Pattern Recognition Vol.9, No.6 (2016), pp.393-406.
[14] Ling $\mathrm{Hu}$ and Qiang Ni, Senior Member, IEEE,'IoTDriven Automated Object Detection Algorithm for Urban Surveillance Systems in Smart Cities" 23274662 (c) 2016 IEEE

[15] Bharath Kumar Perumalla, M. Sunil Babu,,"An Intelligent Traffic and Vehicle Monitoring System using Internet of Things Architecture" Paper ID: ART20162875Volume 5 Issue 11, November 2016 www.ijsr.net

[16] Chandana K K, Dr. S. Meenakshi Sundaram, Cyana D'sa, Meghana N Swamy, Navya K,"“A Smart Traffic Management System for Congestion Control and Warnings Using Internet of Things (IoT)"Saudi J. Eng. Technol.; Vol-2, Iss-5(May, 2017):192-196

[17] P. Pyykönen, J. Laitinen, J. Viitanen,'IoT for Intelligent Traffic System"978-1-4799-14944/13/\$31.00 (C2013 IEEE 175

[18] Misbahuddin, Junaid Ahmed Zubairi, Abdulrahman Saggaf, Jihad Basuni, Sulaiman A-Wadany and Ahmed Al-Sofi,"IoT Based Dynamic Road Traffic Management for Smart Cities"978-1-4673-92686/15/\$31.00 @2015 IEEE

[19] Alisha Janrao, Mudit Gupta, Divya Chandwani, U. A. Joglekar, " Real Time Traffic Density Count using Image Processing", International Journal of Computer Applications (0975 - 8887) Volume 162 - No 10, March 2017

[20] Pratishtha Gupta, G.N Purohit, Amrita Dadhich ," Approaches for Intelligent Traffic System: A Survey" ISSN : 0975 -3397, Vol. 4 No. 09 Sep 2012

[21] Zhiyong Liu," A Survey of Intelligence Methods in Urban Traffic Signal Control", IJCSNS International Journal of Computer Science and Network Security, VOL.7 No.7, July 2007

[22] Shruthi K R and Vinodha K," Priority Based Traffic Lights Controller Using Wireless Sensor Networks" International Journal of Electronics Signals and Systems (IJESS) ISSN: 2231- 5969, Vol-1 Iss-4, 2012

[23] Prof. Latha Venkatesan, S.Omar Farooq, J.Faisal Imraan, K.Jegan Kumar, J.Naveen Kumar," Animals and Vehicle Collision Avoidance Using Wireless Sensor Actuator Network", International Journal of Scientific \& Engineering Research, Volume 4, Issue 5, May-2013 1856 ISSN 2229-5518

[24] Naveen Chintalacheruvu, Venkatesan Muthukumar," Video Based Vehicle Detection and Its Application in Intelligent Transportation Systems", Journal of Transportation Technologies, 2012, 2, 305-314

[25] Hasan Omar Al-Sakran," Intelligent Traffic Information System Based on Integration of Internet of Things and Agent Technology" (IJACSA) International Journal of Advanced Computer Science and Applications, Vol. 6, No. 2, 2015 


\section{BIOGRAPHIES}

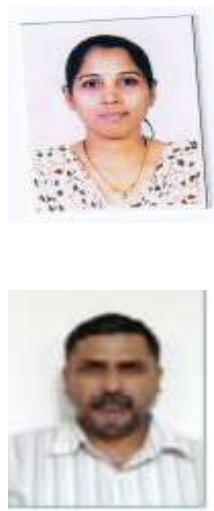

Miss Rashmi P. Nimkar, (M.E. Digital Electronics ) is a student of Prof Ram Meghe Institute of Technology \& Research, Badnera-Amravati Maharashtra, India. interest is Communication Network.

Prof. (Dr.) Chandrashekhar N. Deshmukh, Associate Professor in Prof Ram Meghe Institute of Technology \& Research, Badnera-Amravati , Maharashtra, India, having teaching experience of 20 years and Industrial experience of 6 years. His area of 